\title{
A Criterion That Determines Fast Folding of Proteins: A Model Study
}

\author{
Carlos J. Camacho円 \\ Facultad de Física, P. Universidad Católica de Chile, Casilla 306, Santiago 22, Chile \\ D. Thirumalai/2 \\ Institute for Physical Science and Technology, University of Maryland, College Park, MD 20742
}

\begin{abstract}
We consider the statistical mechanics of a full set of two-dimensional protein-like heteropolymers, whose thermodynamics is characterized by the coil-to-globular $\left(T_{\theta}\right)$ and the folding $\left(T_{f}\right)$ transition temperatures. For our model, the typical time scale for reaching the unique native conformation is shown to scale as $\tau_{f} \sim F(M) \exp \left(\sigma / \sigma_{0}\right)$, where $\sigma=1-T_{f} / T_{\theta}, M$ is the number of residues, and $F(M)$ scales algebraically with $M$. We argue that $T_{f}$ scales linearly with the inverse of entropy of low energy non-native states, whereas $T_{\theta}$ is almost independent of it. As $\sigma \rightarrow 0$, non-productive intermediates decrease, and the initial rapid collapse of the protein leads to structures resembling the native state. Based solely on accessible information, $\sigma$ can be used to predict sequences that fold rapidly.
\end{abstract}

PACS numbers: 87.10.+e, 05.70.Fh, 64.60.Cn

\footnotetext{
${ }^{1}$ e-mail: ccamacho@lascar.puc.cl

${ }^{2}$ e-mail: thirum@glue.umd.edu
} 
An apparent puzzle in the protein folding kinetics was raised by Levinthal [1] in late sixties. He argued that since the number of conformations of even a moderate sized protein is astronomically large, it is unlikely that a polypeptide chain can find the lowest free energy conformation (referred to as the native state) in biologically relevant time scales. We note that the time scale in which proteins fold in cells is several (twelve or more) orders of magnitude longer than microscopic time scales. The belief that proteins find the global free energy minima in times on the order of seconds led Levinthal to postulate that there must be "preferred pathways" that direct the folding process. Minimal protein models, which capture some but not all of the features considered to be important in proteins, have in recent years been used to provide plausible resolutions to this seeming paradox [2, 3, 4, 5]. These scenarios have many common elements but differ significantly in detail. The unifying idea that has emerged from these studies is that in order to quantitatively describe folding kinetics (at least in these models) one has to contend with complex energy landscapes.

A few years ago we showed using simple two dimensional lattice models that, in general, quasi

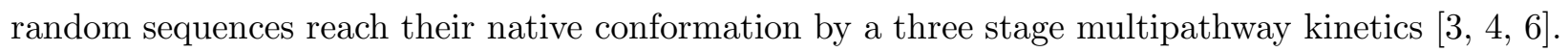
In the first stage the chain collapses from a random coil to a compact state. In the second stage the chain searches among the set of compact structures, in a diffusive reptation like mechanism, to reach one of minimum energy structures. The final stage involves an activated transition from one of the minimum energy structures to the native conformation. Recently tentative estimates of the time scales for the various processes in the three stage kinetics have been suggested in terms of $M$ the number of amino acid residues in a protein and other experimentally controllable parameters [3, 7, 8]. These estimates support our earlier assertion that this three stage kinetics provides a resolution to the Levinthal paradox for single domain proteins which have typically $M$ less than about two hundred. The reason that folding of these single domain proteins occurs in time scales on the order of seconds is that the average free energy barrier in the third stage scales only as $\sqrt{M}$ [7] and not as $M$ as had been supposed by others [9].

With the Levinthal paradox resolved by the three stage multipathway kinetics [3] for quasirandom sequences, it is natural to address the following question: For a given value of $\mathrm{M}$ is there an 
intrinsic property of the sequence that essentially determines the folding time? In this paper we use a simple model, belonging to the class of HP model proposed by Dill and collaborators [10], to answer in affirmative the question raised above. We had conjectured earlier [3] that sequences that fold rapidly are characterized by having the coil-to-globular (collapse) transition temperature, $T_{\theta}$, and the folding temperature, $T_{f}$, in close proximity. In particular, the parameter

$$
\sigma=\left(T_{\theta}-T_{f}\right) / T_{\theta}
$$

can be used to classify kinetic accessibility of the native conformation. In this letter, we present quantitative estimates of folding times in a number of sequences spanning a range of $T_{\theta}$ and $T_{f}$ that explicitly verifies our earlier conjecture. This result implies that kinetic accessibility of the native state may in fact be encoded in the primary sequence of proteins.

The aforementioned prediction can be interpreted in the context of the refolding of a protein-like structure from an unfolded conformation $\left(T>T_{\theta}\right)$ to a folded native-like structure $\left(T \lesssim T_{f}\right)$. It is reasonable to suggest that $\sigma$ probes the key role played by the "molten globular" states in the folding dynamics. (a) For large $\sigma$, the dynamical process involves a detailed sampling of transient globular states in a rough free energy landscape [3, 4, 7, 8], which naturally slows down the folding kinetics. On the other hand, (b) for $\sigma$ small, the collapse and folding occur almost simultaneously, with the chain collapsing almost directly into a folded structure. This was the rationalisation to our earlier prediction [3]: the smaller the value of $\sigma$, the smaller the value of the folding time scale $\tau_{f} !$

The polypeptide chain is modelled as a two letter self-avoiding walk on a two dimensional square lattice [10]. Each bead on the chains can be either hydrophobic (H) or hydrophilic (P). When two non-bonded $\mathrm{H}$ beads are nearest neighbors the interaction is assumed to be $-\varepsilon<0$. All other interactions are zero. The temperatures $T_{\theta}$ and $T_{f}$ are computed exactly using series enumeration of the finite-size chains (see, e.g., [3, 4, 10, 11]). The simulation results have been obtained by using a single step Monte Carlo dynamics and a Metropolis algorithm. All time scales are measured in Monte Carlo steps (MCS). Allowed moves are such that they mimic basic features 
of real chain dynamics, i.e. preserve chain connectivity and excluded volume interactions [12]. Sequences studied are such that the ratio of the number of hydrophobic $M_{H}$ to hydrophilic $M_{P}$ sites is set close to its optimal folding value of one 顿, and they have a unique ground state assumed to be the native state. Thus, we restrict our analysis to (thermodynamically) foldable sequences. To free our statistical analysis from a bias sampling, we consider all possible sequences with the aforementioned properties, and protein sizes equal to $M=15-M_{H}=8$ (214 sequences) and $M=18-M_{H}=10$ (1326 sequences).

The time scale $\tau_{f}$ was measured by fitting and averaging the exponential decay $\left(\exp \left[-t / \tau_{f}\right]\right)$ of the long-time deviation from equilibrium of several correlation functions after a temperature quench from a high temperature unfolded structure to the folding temperature $T_{f}$. For details see Ref. [3]. One of the correlation functions used is the overlap function $\langle\chi(t)\rangle$ which depends on all distances $r_{i j}$ between sites, with $i$ and $j$ indicating site index along the chain. This function is a useful probe of the folding kinetics [3], and is defined as follows

$$
\langle\chi(t)\rangle=1-\frac{1}{M^{2}-3 M+2}\left\langle\sum_{i \neq j, j \pm 1} \delta\left(r_{i j}(t)-r_{i j}^{N}\right)\right\rangle .
$$

It measures structural differences between fluctuating conformations and the ground (or "native") state denoted by the superscript $N$. ( $\langle\chi\rangle$ varies between 1 for a fully non-native structure and 0 for the pure native state. Random overlap of two structures amounts to $\langle\chi\rangle=0.735$ for $M=15$.) We denote non-native states as those structures with, at least, one topological feature different from the native state. The folding temperature $T_{f}$ is defined as the temperature at which the fluctuations $\Delta \chi=\left\langle\chi^{2}\right\rangle-\langle\chi\rangle^{2}$ show a peak. Below $T_{f}$ conformations are mostly native, whereas above $T_{f}$ they are non-native. For a protein model with short range interactions, the standard definition for $T_{\theta}$ is the temperature at which the energy fluctuations $\left(\left\langle E^{2}\right\rangle-\langle E\rangle^{2}\right)$ peak, these fluctuations are trivially related to the specific heat which were used to define $T_{\theta}$ in Ref. [3]. For the cases cited in [3], $\sigma \approx(0.50,0.63,0.088)$, whereas $\tau_{f} \approx(40,230,1) \times 10^{5}$ MCS, for models A, B and $\mathrm{C}$, respectively. This apparent correlation has also been recently observed in three dimensional lattice simulations by Socci and Onuchic [13]. These authors suggested that reducing the average 
energetic drive toward compactness may lead to a smaller difference in $T_{\theta}-T_{f}$.

The first correlation of interest is between $\sigma\left(T_{\theta}, T_{f}\right)$ and the folding time scale $\tau_{f}$. Figure 1 summarizes this analysis as a function of the parameter $\sigma$, showing: (a) histograms of $\sigma$ for the space of sequences mentioned above; (b) the average overlap of the first exited states with respect to the ground state $\langle\chi\rangle_{1 s t}$; and (c) $\tau_{f}$ (the equilibration time scale at $T_{f}$ ) for 30 random sequences with $M=15$. (a) The histograms of $\sigma$ show broad distributions between $\sigma \simeq 0.2$ and 0.7 . (b) The average resemblance of the metastable states $\left(1^{\text {st }}\right.$ exited states) with the native structure decreases as $\sigma$ increases. Indeed, for large $\sigma$ the overlap is close to that of random structures. Hence, as $\sigma$ increases metastable states are further apart in configurational space. (c) Based on the above, it is not surprising to find that $\tau_{f}$ varies by almost three orders of magnitude as a function of $\sigma$. We find the scaling

$$
\tau_{f} \simeq F(M) \exp \left[\sigma\left(T_{\theta}, T_{f}\right) / \sigma_{0}\right]
$$

which we predict to be universal. Although it was not checked here, we expect the prefactor $F(M) \sim M^{\lambda}$ to also be a universal scaling function with $\lambda \simeq 3$, note the resemblance with Eq. 4 of Ref. [8], and Refs. [3, 7, 14]. The constant $\sigma_{0} \simeq 0.11$ is a model dependent parameter. The most important conclusion of this analysis is that fast folding sequences are characterized by having small values of $\sigma$. Some limited experimental verification of this prediction have recently been shown on fast folding cytochrome $c$ [15], where folding and collapse have been found to be almost synchronous.

Since folding times correlates well with $\sigma$ it is instructive to find a relationship between $\sigma$ and the energy spectrum. Theoretically, we expect $\mathcal{F}_{N}\left(T_{f}\right) \approx \mathcal{F}_{N N}\left(T_{f}\right)$, where $\mathcal{F}$ denotes free energy and $N$ and $N N$ stand for native and non-native states, respectively. Hence, one can write the following equation

$$
T_{f} \approx\left(\left\langle U_{N N}\right\rangle-\left\langle U_{N}\right\rangle\right) /\left(\left\langle S_{N N}\right\rangle-\left\langle S_{N}\right\rangle\right),
$$

where $\langle U\rangle$ and $\langle S\rangle$ denote internal energy and entropy. At $T_{f}$ it is reasonable to assume that the leading contribution to the statistical averages come largely from the low-lying states. Thus, we 
can estimate $\left\langle U_{N N}\right\rangle-\left\langle U_{N}\right\rangle \sim \varepsilon$, obtaining

$$
\varepsilon / k_{B} T_{f} \approx \ln \left(\Omega_{N N} / \Omega_{N}\right)
$$

where $\Omega$ corresponds to the number of states with $\Omega_{N} \sim 2$ (i.e. ground state plus its mirror image), and $k_{B}$ is the Boltzmann constant hereafter set to one. We conclude that $T_{f}$ must depend linearly on the "entropy" of non-native states. We expect (5) to be an excellent estimate for those sequences with a sparse low energy spectrum.

This prediction is in very good agreement with Fig. 2, where we show $\varepsilon / T_{f}$ as a function of the degeneracy of the first $\Omega_{1}$, second $\Omega_{2}$ and third $\Omega_{3}$ exited states. For degeneracies larger than $30, \varepsilon / T_{f}$ scales almost linearly with the logarithm of $\Omega_{1} / 2$, see solid line in Fig. 2 . The striking agreement between the fit and (5) led us to conclude that $\Omega_{N N} \simeq \Omega_{1}$ for large $\Omega_{1}$. Although noisier a similar correlation is observed for higher energy levels as well. Degeneracies of the energy levels grow exponentially, on an average, by a model dependent factor of the order of 10 as the energy increases by one. Clearly, this factor depends on the physical constraints of the connectivity between states. These observations suggest a certain hierarchy and organization of the energy landscape, with closely related energy levels. These correlations can be model dependent.

Also shown in Fig. 2 is the small but definite dependence of the inverse collapse temperature $\varepsilon / T_{\theta}$ with $\Omega_{1}$. Fast folding sequences have a somewhat lower $T_{\theta}$, suggesting that these sequences have a smaller energetic drive toward compactness [13]. Figure 2 shows the link between the broadening of $T_{\theta}-T_{f}$ and "molten globular" states. For clarity, we have also plotted the histograms for $\Omega_{1}$ whose shapes are reminiscent of those in Fig. 1. As the ratio $M_{H} / M_{P}$ deviates from unity, the number of exited states increases dramatically 画, with $\sigma$ and $\tau_{f}$ increasing accordingly. Hence, this analysis presents further evidence regarding the role played by intermediate states $(\Omega)$ in the folding dynamics, and the natural selection of proteins with an optimum content of hydrophobic residues [4].

It is noteworthy that the energy gap between the native state and metastable states in this model is always $\varepsilon$. A trivial check of correlations between $\varepsilon$ and $\tau_{f}$ shows that there is none. This, 
of course, contradicts an earlier suggestion of Šali, Shahknovich and Karplus [16] who suggested that the energy gap was enough to predict chain "foldicity", or ease to fold. The gap appropriately divided by $T_{f}$ does seem to show a correlation with $\tau_{f}$ [17]. This correlation, however, deteriorates for fast folding sequences governed by entropy [18].

Goldstein et. al. 19 proposed that rapid folding sequences are characterized by having a large value of the ratio $T_{f} / T_{g}$, where $T_{g}$ is an equilibrium transition temperature. From a practical point of view, however, this prediction which appears to be supported by simulations [20], provided $T_{g}$ is replaced by a kinetic glass transition temperature, is not very useful. Indeed, there is no straightforward technique to measure the glass transition temperature, other than to estimate $T_{g}$ from a detailed knowledge of the unknown $\tau_{f}$.

It is quite clear that based on two thermodynamic parameters one cannot fully describe the folding dynamics in a complex energy landscape. The above notwithstanding, one can establish meaningful statistical relationships between equilibrium properties and a given set of dynamical rules. As long as these rules mimic essential features of the physical processes, the relationships should shed some light on the underlying mechanisms.

We have shown that experimentally accessible information, namely $\sigma=1-T_{f} / T_{\theta}$ where $T_{\theta}$ is the coil-to-globular and $T_{f}$ is the folding transition temperature, could be used to predict and design fast folding sequences of proteins. For sequences that fold rapidly we predict that folding times $\tau_{f}$ should scale as suggested by (3). This expression embodies the interplay between energy frustration and entropic barriers. It recovers the slow folding limit when the protein size $M$ increases and few sequences fold fast. Our postulates are physically limited by its statistical nature. In particular, averaging over sequence randomness entails standard deviations in $\tau_{f}$ of the order of half a decade. From a microscopic point of view, $\sigma$ appears to be related to entropy of low-lying states. Minimally frustrated fast folding sequences with relatively small $\sigma$ fold in time scales mostly governed by entropic considerations.

This work was supported in part by a grant from the National Science Foundation (CHE93- 
07884) and the Air force office of Scientific Research (F496209410106). CJC acknowledges support from FONDECYT No. 3940016 (Chile). 


\section{References}

[1] Levinthal C., in Mossbauer Spectroscopy in Biological Systems, edited by P.Debrunner, J.C.M. Tsibris and E. Münck, E, (University of Illinois Press, Urbana) 1968.

[2] Bryngelson J.D. and Wolynes P.G. Proc. Natl. Acad. Sci. USA, 84 (1987) 7524.

[3] Camacho C.J. and Thirumalai D., Proc. Natl. Acad. Sci. USA, 90 (1993) 6369,

[4] Camacho C.J. and Thirumalai D., Phys. Rev. Lett., 71 (1993) 2505.

[5] Shakhnovich E., Phys. Rev. Lett., 72 (1994) 3907.

[6] Camacho C.J. and Thirumalai D., Proc. Natl. Acad. Sci. USA, 90 (1995) 1277.

[7] Thirumalai D., J. de Phys. I (Paris), 5 (1995) 1457.

[8] Camacho C.J., Entropic barriers, Frustration and Order: Basic Ingredients in Protein Folding, cond-mat/9512019, to be published.

[9] Karplus M. and Šali A., Curr. Opin. Struct. Biol., 5 (1995) 58.

[10] Lau K.F. and Dill K.A. Macromolecules, 22 (1989) 3986; Chan H.S. and Dill K.A. ibid 22 (1989) 4559.

[11] Shakhnovich E. and Gutin A.M., J. Chem. Phys., 93 (1990) 5967.

[12] Verdier P.H., J. Chem. Phys., 59 (1973) 6119.

[13] Socci N.D. and Onuchic J.N., J. Chem. Phys. 103 (1995) 4732.

[14] Thirumalai D., in Statistical Mechanics, Protein Structure and Protein Interactions, edited by S. Doniach (Plenum, N.Y.) p. 115-134 (1994).

[15] Sosnick T.R., Mayne L., Miller R. and Englander S.W., Nature Struct. Biol., 1 (1995) 149.

[16] Šali A., Shakhnovich E. and Karplus M., Nature 369 (1994) 248. 
[17] Gutin A.M., Abkevich V.I., and Shakhnovich E.I., Proc. Natl. Acad. Sci. USA, 92 (1995) 1282.

[18] C.J. Camacho and D. Thirumalai, Proteins: Struct. Funct. Genet. 22 (1995) 27.

[19] Goldstein R., Luthey-Schulten Z. and Wolynes P.W., Proc. Nat. Acad. Sci. USA, 89 (1992) 4918.

[20] See, e.g., Socci N.D. and Onuchic J.N., J. Chem. Phys., 101 (1994) 2. 


\section{Figure Captions}

1. Statistical analysis of $\sigma$ for non-degenerate sequences with $M=15$ and $M=18$ (see text). (a) Bottom, histograms of $\sigma$ : dashed line and solid lines correspond to $M=15$ and $M=18$, respectively. (b) Middle, average overlap between all first exited states and the ground state $\langle\chi\rangle_{1 s t}:+$ and $\diamond$ symbols correspond to $M=15$ and $M=18$, respectively. Least square fit shows a linear dependence of $\langle\chi\rangle_{1 s t}$ on $\sigma$ (solid line). (c) Top, folding time scale $\tau_{f}$ ( $\mathrm{a}$ ) as a function of $\sigma$ for 30 random sequences with $M=15$. Error bars are of the order of symbol size. Least square fit yields $1.8 \times 10^{5} \exp (\sigma / 0.11)$ (solid line). Bars in (b) and (c) correspond to one standard deviation.

2. Inverse of folding temperature as a function of the number of first $\left(\Omega_{1}\right)$, second and third exited states. Solid line corresponds to $\varepsilon / T_{f}=\ln \left(\Omega_{1} / 2\right)$. Symbols are as in Fig. 1b. To better resolve the overlap of symbols, we show histograms of $\Omega_{1}$ for $M=15$ (dashed line) and $M=18$ (solid line). Also shown is inverse of collapse temperature $\varepsilon / T_{\theta}(\times)$ as a function of $\Omega_{1}$. 